\title{
Bipartite Graphs as Models of Complex Networks
}

\author{
Jean-Loup Guillaume and Matthieu Latapy \\ LIAFA - CNRS - Université Paris 7 \\ 2 place Jussieu, 75005 Paris, France. \\ (guillaume,latapy)@liafa.jussieu.fr
}

\begin{abstract}
It appeared recently that the classical random graph model used to represent realworld complex networks does not capture their main properties. Since then, various attempts have been made to provide accurate models. We study here a model which achieves the following challenges: it produces graphs which have the three main wanted properties (clustering, degree distribution, average distance), it is based on some realworld observations, and it is sufficiently simple to make it possible to prove its main properties. This model consists in sampling a random bipartite graph with prescribed degree distribution. Indeed, we show that any complex network may be viewed as a bipartite graph with some specific characteristics, and that its main properties may be viewed as consequences of this underlying structure. We also propose a growing model based on this observation.
\end{abstract}

\section{Introduction.}

It has been shown recently that most real-world complex networks have some specific properties in common. These properties are not captured by the model generally used before this discovery, although they play a central role in many contexts like the robustness of the Internet [6, 15, 19, 20, 49], the spread of viruses or rumors over the Internet, the Web or other social networks [48, 52, 58, the performance of protocols and algorithms [36, 41, 68], and many other.

This is why a strong effort has been put in the realistic modeling of complex networks in the last few years, and much progress has been accomplished in this field. Some models achieve the aim of producing graphs which capture some, but not all of the main properties of real-world complex networks. Some models obtain all the wanted properties but rely on artificial methods which give unrealistic graphs (trees, graphs with uniform degrees, etc). Others rely on construction processes which may induce some hidden properties, or are too difficult to analyze.

In this paper, we propose the random bipartite graph model as a general model for complex networks. We will show that this model produces graphs with many observed properties. It relies on real-world observations and gives realistic graphs. Finally, it is simple enough to make it possible to prove its main properties. We will also discuss some identified drawbacks of this model.

We will first present an overview of the context in which our work lies. In particular, we use some ideas introduced in previous papers, which we need to describe precisely. Then 
we show how all complex networks may be described as bipartite structures. After this, we present the random bipartite model and analyze it to show that the main properties of complex networks are somehow a consequence of their underlying bipartite structure. We also present a growing bipartite model based on the same ideas. Finally we discuss the advantages and limitations of these models.

\section{Context.}

Throughout our presentation, we will use a representative set of complex networks which have received much attention and span quite well the variety of contexts in which complex networks appear: an Internet graph at router level [16, 32, 33, consisting of physical links between routers ; a web graph from Notre Dame university [5, 23] where web pages are linked by hyperlinks ; a co-occurence graph in which words are linked if they belong to a same sentence in a given text [31, 67] ; the actor graph where actors are linked when they have played together [24, 70]; a co-authoring graph from Arxiv [7] where scientists are linked if they have signed a paper together [50, 51, 56]; and finally a protein graph where two proteins of a given biological system are linked if they influence each other [23, 35].

Many other complex networks have been studied. Refer to [4, 26, 54, for a more descriptive list of networks and corresponding references. All these networks have some properties in common which have been discovered quite recently and have concentrated a large attention in various communities. Hereafter we present the properties in concern and some recent efforts in the modeling of these properties.

\subsection{Statistical properties}

Most real-world complex networks have a number of edges $m$ which scales linearly with the number of vertices $n: m \sim k \cdot n$ where $k$ is the average degree (which does not depend on the size of the graph). Therefore, these networks have a low density (going to 0 when $n$ grows), the density being defined as the number of existing edges over the number of edges that could exist $(n(n-1) / 2)$.

The distance between two vertices, defined as the number of edges on a shortest path between these vertices, is low on average. It is a well known property on social networks since the work of Stanley Milgram [43] and the notion of "six degrees of separation". However it appeared more recently that all complex networks have a low average distance which typically scales like the logarithm of the size of the graph. It has been shown that this is actually true for any graph which contains some reasonable amount of randomness. Actually, under reasonable assumptions, the average distance in random graphs scales even slower than the logarithm 1 of their size [13, 18, 21, 28, 39, 55, 56].

The local clustering, or clustering coefficient [70, is defined for each vertex of degree at least 2 as the proportion of edges between its neighbors: $c(u)=\frac{|\{(x, y), x, y \in N(u)\}|}{\left(\begin{array}{c}d(u) \\ 2\end{array}\right)}$, where $d(u)$ is the degree of vertex $u$ and $N(u)$ is the set of neighbors of $u$. The clustering of a graph is simply the average over all vertices. All real-world complex networks have a high clustering which seems to be independent of the size of the network. These graphs are locally dense while globally sparse.

\footnotetext{
${ }^{1}$ Typically like $\log (n) / \log (\log (n))$.
} 


\begin{tabular}{|l|c|c|c|c|c|c|}
\hline & Internet & Web & Actors & Co-auth & Co-occur & Protein \\
\hline \hline$n$ & 75885 & 325729 & 392340 & 16401 & 9297 & 2113 \\
\hline$m$ & 357317 & 1090108 & 15038083 & 29552 & 392066 & 2203 \\
\hline density & $1.2 \mathrm{e}-4$ & $2.1 \mathrm{e}-5$ & $1.9 \mathrm{e}-4$ & $2.2 \mathrm{e}-4$ & $9.1 \mathrm{e}-3$ & $9.9 \mathrm{e}-4$ \\
\hline$c$ & 0.171 & 0.466 & 0.785 & 0.638 & 0.822 & 0.153 \\
\hline$\alpha$ & 2.5 & 2.3 & 2.2 & 2.4 & 1.8 & 2.4 \\
\hline$d$ & 5.80 & 7 & 3.6 & 7.18 & 2.13 & 6.74 \\
\hline
\end{tabular}

Table 1: The main statistics for the complex networks we use in this paper. For each network, we give its number of vertices $n$, its number of links $m$, its density, the value of the exponent $\alpha$ of the power law that fits best its degree distribution, its clustering $c$, and its average distance $d$.

Finally, the degree distribution which is, for each $k$, the probability $p_{k}$ that a randomly chosen vertex has degree $k$, is completely different from what was expected. Indeed for almost all real-world complex networks, the degree distribution follows a power law: $p_{k} \sim k^{-\alpha}$, while one would have expected an exponential decrease (Poisson-like distributions). The exponent $\alpha$ of the power law is generally between 2 and 3. Such a distribution means that although most vertices have a small degree, the number of vertices with degree $k$ decays only polynomially with $k$, and therefore there is a significant number of vertices with high degree.

The main properties of the real-world complex networks we use in this paper are given in Table 1. Notice that, as announced, all these real-world complex networks have a very low density, a low average distance, a power law distribution of degrees and a high clustering.

The similarity of these networks concerning unexpected properties has led to the study of other properties. The simplest one concerns the degree-degree correlation: what is the average degree of the neighbors of a vertex of degree $k$. Three main behaviors are expected, either high-degree vertices tend to connect to high-degree vertices, or to low-degree vertices, or to any nodes. These behaviors can be observed using the the slope (increasing, decreasing or constant) of the plot which relates the average degree of the neighbors of nodes of degree $k$, to $k$ [11, 63, 64], or with a single parameter (assortativity coefficient), which may be positive (assortative networks), negative (dissortative networks) or null (neutral networks) [53]. Most social networks are assortative (similar vertices are connected) while technological or biological are generally dissortative [53].

One may also correlate the clustering and the degree by computing the average clustering of vertices with a given degree. This also defines assortative (high degree yields high clustering), neutral or dissortative networks.

Finally, other properties have been studied, such as the centrality (how many shortest paths contain a given vertex) [51, the distribution of eigenvalues of the adjacency matrix [30, 42], etc. All these statistical properties are used to describe a given complex network and to study the similarities and differences between several complex networks. They give precise insight on what one may expect when considering a complex network having a set of properties. 


\subsection{Modeling complex networks}

The basic model for complex networks is the Erdös-Rényi (ER) random graph model [13, 29]. In a random graph with $n$ vertices, each of the $\frac{n \cdot(n-1)}{2}$ possible edges exists with a given probability $p$ (this model is know as $\mathcal{G}_{n, p}$ ). The average distance of $\mathcal{G}_{n, p}$ scales with the logarithm of $n$ [13. Moreover, the clustering is equal to the connection probability $p$ since each pair of vertices is connected with the same probability independently of the fact that they are both linked to a same vertex. If $m \sim k \cdot n$ as in real-world complex networks, this means that the clustering scales as $n^{-1}$ and therefore tends to 0 when $n$ grows. Finally, the degree distribution follows a Poisson law $p_{k} \sim e^{-\lambda} \frac{\lambda^{k}}{k !}$ [13], which implies in particular that all vertices have nearly the same degree.

Therefore, although this model can be considered as relevant concerning the average distance, it misses two main properties of real-world complex networks. In particular, the degree distributions are qualitatively different.

It is however possible to sample uniformly a random graph with a given degree distribution (in particular a power law) [10, 12, 40, 45, 46] using the configuration model, or Molloy and Reed2 (MR) model: for each vertex, draw its degree at random according to the given distribution, create as many connection points as its degree and finally connect pairs of connection points at random 3 .

The power law graphs obtained this way have an average distance which scales slower that the logarithm of their size [18, 21, 28, 39, 55, 56. Moreover, the fact that vertices are linked together purely at random (only their number of edges is given) makes it possible to study the properties of the obtained graphs, and it indeed seems that it captures some of the most important behaviors of complex networks [2, 18, 39, 54, 58, However, under reasonable assumptions on the degree distribution, the clustering of these graphs tends to zero when $n$ grows [54].

This approach could in principle be continued to sample a graph among the ones having a given number of nodes, a given degree distribution and a given clustering. However, until now, there is no known method to sample uniformly such a graph, and the problem seems difficult.

On the other hand, a large variety of models based on the iteration of a construction process inspired from the way complex networks grow in reality have been introduced.

The first generic model of real-world complex networks, has been introduced in 1998 by Watts and Strogatz (WS) [70. One starts with a ring of $n$ vertices in which each vertex is connected to its $k$ nearest neighbors, for a given $k$. Then, each edge is rewired with a given probability $p$ by choosing randomly a new extremity. When $p$ is small the graph is almost a ring which have high average distance and high clustering. On the opposite, when $p$ is high, the graph is nearly random. For medium values of $p$, the graph has both a small average distance and a high clustering, but the degree distribution of the obtained graphs does not follow a power law [25, 70].

\footnotetext{
${ }^{2}$ Despite it has been introduced in [10] and studied in [12, this model is often referred to as the Molloy and Reed model since these authors made it popular in their contributions [45, 46]. We will follow this convention here.

${ }^{3}$ Note that this algorithm may induce multiple links and loops. One may also use techniques to avoid them, see for instance [38, 44, but this is out of the scope of this paper.
} 
Another important step was done with models based on preferential attachment. [3, 27, 37. The "rich gets richer" principle can be derived in a model where vertices arrive one by one in a graph and choose their neighbors with a probability proportional to the degree of these neighbors. This model has been studied intensively and is now well known [4: the degree distribution follows a power law with exponent, the average distance is logarithmic in the number of vertices, and the clustering is low, going to 0 when the number of vertices grows. Despite this last point, this model has received much attention, in particular because it defines growing graphs.

Both the WS model and the AB one have been introduced to model generic behavior of complex networks. If they both fail in producing graphs having each of the three properties we cited, they have been widely used as building blocks for more complex models.

Table 2 shows the performances obtained with the basic models we cited in our practical cases. Let us insist on the fact that the models seek qualitative properties (non negligible clustering, power law degree distribution, etc). Their aim is not to produce graphs with exactly given values for these properties. However, even with this in mind, the graphs obtained using these models are significantly different from real-world ones concerning at least one of these three points.

\begin{tabular}{|l|c|c|c|c|c|c|}
\hline & Internet & Web & Actors & Co-auth & Co-occur & Protein \\
\hline \hline$n$ & 75885 & 325729 & 392340 & 16401 & 9297 & 2113 \\
\hline$m$ & 357317 & 1090108 & 15038083 & 29552 & 392066 & 2203 \\
\hline$c$ & 0.171 & 0.466 & 0.785 & 0.638 & 0.822 & 0.153 \\
\hline$c_{E R}$ & 0.0001 & 0.00002 & 0.0002 & 0.0002 & 0.009 & 0.001 \\
\hline$c_{M R}$ & 0.0694 & 0.017 & 0.0057 & 0.001 & 0.26 & 0.007 \\
\hline$c_{A B}$ & 0.0024 & 0.0005 & 0.0015 & 0.003 & 0.028 & 0 \\
\hline$c_{W S}$ & 0.171 & 0.461 & $0.74\left(^{*}\right)$ & $0.523\left(^{*}\right)$ & $0.74\left(^{*}\right)$ & $0.06\left(^{*}\right)$ \\
\hline \hline$d$ & 5.80 & 7 & 3.6 & 7.18 & 2.13 & 6.74 \\
\hline$d_{E R}$ & 5.25 & 5.47 & 2.97 & 7.57 & 2.06 & 10.4 \\
\hline$d_{M R}$ & 3.25 & 4.48 & 2.95 & 5.77 & 2.36 & 5.73 \\
\hline$d_{A B}$ & 4.15 & 5.1 & 2.93 & 5.5 & 2.38 & 8.15 \\
\hline$d_{W S}$ & 5.90 & 11.23 & $2559\left(^{*}\right)$ & $2269\left(^{*}\right)$ & $55.6\left(^{*}\right)$ & $509\left(^{*}\right)$ \\
\hline
\end{tabular}

Table 2: Performance of the main generic models for complex networks. For each network, we give its number of vertices $n$, its number of links $m$, its clustering $c$, and its average distance $d$. Moreover, we give the values of these parameters for typical graphs with the same number of vertices and edges obtained with ER, MR, AB and WS models. Moreover, in The cases pointed by a star $\left(^{*}\right)$, the real clustering is too large to be obtained with the WS model. Therefore we used in these cases the parameters inducing the maximal clustering, which yields very large average distances.

Many other attempts have been made to reach the goal of obtaining models which give graphs having each of the three main properties we have cited. Most of them are described in [4, 26, 62]. Some deterministic models, which we do not detail here, have also been introduced [8, 22] which produce the wanted properties and are suitable for analysis. However, they cannot be considered as realistic and the obtained graphs have specific properties which make them very different from real-world complex networks. 
Another model is the Dorogovstev and Mendes (DM) model which generates highly clusterised graphs with a power law degree distribution [26]. In this model, at each step a vertex is created, an edge is chosen at random and the new vertex is connected to both extremities of the edge. Since high-degree vertices have more edges, they are more likely to be chosen. The hidden preferential attachment and the creation of triangles induces the wanted properties. However the parameters of this model cannot be tuned and it has some unexpected properties (for instance, there is no node of degree 1 and it produces planar graphs 4 ). Therefore we are not going to use it hereafter.

More recent models [9, 59, 60, 61, 65, 66, 69] have been introduced. Some of them use the preferential attachement principle, and create some triangles at each step. In [59], for each newly created edge, a triangle is built using both extremities of this edge and a neighbor of the old vertex. In [69, a step of the algorithm consists either in the creation of a new vertex with $m$ edges or in the creation of $m$ edges between neighbors of preferentially chosen vertices.

The model introduced in [60] is an evolution of the configuration model in which both the degree distribution and the clustering distribution can be pre-defined. Connections points are created for each vertex in the future network and a first step consists in creating triangles using these connections points. A second step closes the graph by connecting unused connections.

In this paper, we propose a solution to the random sampling of graphs which have all the three wanted properties. To achieve this, we focus on another property of all real-world complex networks, namely their underlying bipartite structure, in Section 2. We then propose two models: the random sampling of bipartite graphs with prescribed degree distributions, and the growing bipartite model with preferential attachment in Section 3. Indeed, as shown in Sections 4 and 5, respectively formally and experimentally, these models induce the three wanted properties. This means that they can be viewed as consequences of the underlying bipartite structure of all complex networks, which is our main contribution.

\section{Complex networks as bipartite graphs}

A bipartite graph is a triple $G=(\top, \perp, E)$ where $\top$ and $\perp$ are two disjoint sets of vertices, respectively the top and bottom vertices, and $E \subseteq \top \times \perp$ is the set of edges. The difference with classical graphs lies in the fact that edges exist only between top vertices and bottom vertices.

Two degree distributions can naturally be associated to such a graph, namely the top degree distribution: $\top_{k}=\frac{|\{t \in \mathrm{T}: d(t)=k\}|}{|T|}$ and the bottom degree distribution: $\perp_{k}=\frac{|\{t \in \perp: d(t)=k\}|}{|\perp|}$. These two distributions play a central role in the following.

\section{Natural bipartite structures}

As already noticed for instance in [34, 55, some complex networks display a natural bipartite structure. Among our examples, one can view Actors (two actors are linked if they are part of a same cast) as a bipartite graph where $\top$ is the set of movies, $\perp$ is the set of actors, and each actor is linked to the movies he/she played in. Coauthoring can also be viewed this way

\footnotetext{
${ }^{4} \mathrm{~A}$ graph is planar iff it can be embedded in the plane so that no edges intersect. Since edges are transformed in triangles, one can easily be convinced that one step of the algorithm does not create edges intersections.
} 
with $T$ being the set of papers and $\perp$ being the set of authors, each author being linked to the papers he/she (co-)signed. Likewise, in Cooccurrence one can link each sentence to the words it contains.

Given a bipartite graph $G=(\top, \perp, E)$, one can easily obtain its classical version, also called the one-mode or $\perp$-projection, defined as $G^{\prime}=\left(\perp, E^{\prime}\right)$ where $\{u, v\}$ is in $E^{\prime}$ if $u$ and $v$ are both connected to a same (top) node in $G$. See Figure 1 for an example. From the bipartite versions of Actors, Coauthoring and Cooccurrence graphs, one can then recover their classical versions. In the $\perp$-projection of a bipartite graph, each top vertex induces a clique (complete subgraph) between the bottom vertices to which it is linked: all actors of a given movie have played together therefore they must be all linked.

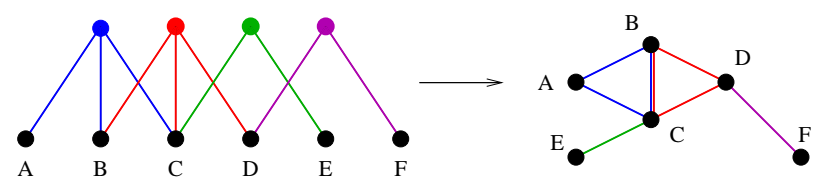

Figure 1: A bipartite network and its $\perp$-projection. Notice that the link $\{B, C\}$ is obtained twice since $B$ and $C$ have two neighbors in common in the bipartite network.

However, given the $\perp$-projection of a bipartite graph, it is in general not possible to recover the bipartite graph from which it has been obtained in an unique way. Similarly if a graph is not naturally bipartite there may exist many bipartite versions of it.

\section{Recovering a bipartite structure}

For the sake of completeness, we now recall and detail the decomposition scheme we proposed in 34] which, given a graph, produces a bipartite graph whose $\perp$-projection is the initial graph. The aim of this scheme is to obtain a bipartite graph whose properties are similar to the ones met in natural bipartite graphs, namely the number of top vertices has the same order of magnitude as the number of bottom vertices and there are some high-degree top nodes (see below and Figure 5).

First notice that the decomposition scheme is nothing but a clique covering problem: it computes a set of cliques (which will correspond to the top nodes in the bipartite graph) such that each edge belongs to at least one clique (which ensures that the $\perp$-projection of the decomposition is exactly the original graph). Simple ideas to cover the graph with cliques might be to consider each edge as a clique, or to consider all maximal cliques. However, the first approach would not yield large cliques while the second one could yield too many cliques (the number of maximal cliques may be exponential).

To reach our goal, we proposed [34] the following decomposition. We pick for each edge a largest clique containing it: a clique whose size is maximal among the ones containing the edge. Notice that this clique may contain only two vertices. Moreover, if there are several such cliques for the same edge, we pick one at random. This decomposition ensures the complete covering of the graph. Moreover, the number of cliques is at most equal to the number of edges, which is of the order of the number of vertices. Finally, since we take largest cliques, we expect to find most of the large cliques contained in the graph.

In the case of Figure 1 we obtain several cliques of size 2 (namely $\{C, E\}$ and $\{D, F\}$ ), and we have to choose at random between $\{A, B, C\}$ and $\{B, C, D\}$ when considering the 
edge $\{B, C\}$. However, these two cliques are obtained from other edges, and we finally obtain a unique decomposition which is nothing but the bipartite graph on the left of the figure.

The central aim of our decomposition scheme is, given the $\perp$-projection of a natural bipartite graph, to produce an artificial bipartite graph similar to the original bipartite graph itself. A way to evaluate it is therefore to decompose the $\perp$-projection version of a natural bipartite complex network and to compare the obtained bipartite network to the original one. This is what we do in Figure 2 ,
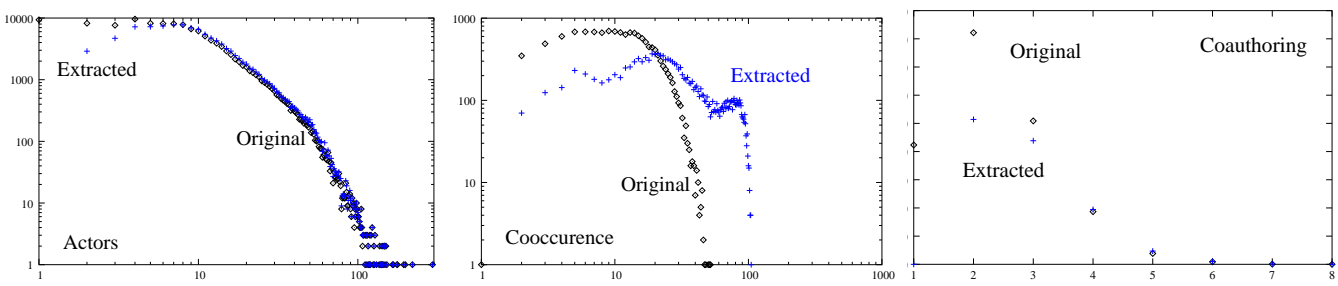

Figure 2: Original clique size distribution for Actors, Cooccurrence and Coauthoring, and extracted clique sizes distribution with the decomposition scheme.

The obtained distributions display some differences for the three graphs decomposed. First, the decomposition scheme produces no cliques of size 1 since the smallest extracted element is the edge (a 2-clique). Moreover, many 2-cliques have not been found, which means that these 2-cliques are not maximal in the original graph. For cliques of size more than 2, our extraction algorithm has been able to find most cliques, or even more. Such new large cliques are induced by the overlapping of other cliques. Notice that in the case of Cooccurrence there are many new very large cliques which have been created by overlapping, while in Actors and Coauthoring this phenomena is very weak. We will discuss more about the overlapping in the conclusion. Despite these differences, the obtained size distributions are similar to the original ones in the sense that we obtain a nontrivial number of large cliques and a similar number of cliques, which are the two main points here.

\section{Practical computation}

Notice that minimizing the number of cliques leads to the minimal clique covering problem which is known to be NP-complete [47, 57]. Computing maximal cliques of a graph is also NP-complete [1, 14] and so is the computation of the largest clique containing a given edge $\{u, v\}$. However, some heuristics make it possible to compute it if the graph is not too large. In our case, we use the following remarks. Let us denote the sets of neighbors of a vertex and an edge by $N(u)=\{v \in V \mid\{u, v\} \in E\}$ and $N(u, v)=N(u) \cap N(v)$ respectively. The largest clique containing $\{u, v\}$ in $G$ is $\mathcal{C} \cup\{u, v\}$, where $\mathcal{C}$ is the largest clique in the sub-graph of $G$ induced by $N(u, v)$. Figure 3 illustrates this process.

Recall that the decomposition process relies on a NP-complete problem in general. However, we observed that in real-world complex networks, the sub-graphs induced by $N(u, v)$ for all edges $\{u, v\}$ are in general very dense and very small (Figure 44), which is due to the high clustering and to the power law degree distribution, respectively. The small size makes it possible to compute the largest clique containing $\{u, v\}$ very efficiently in practice. 


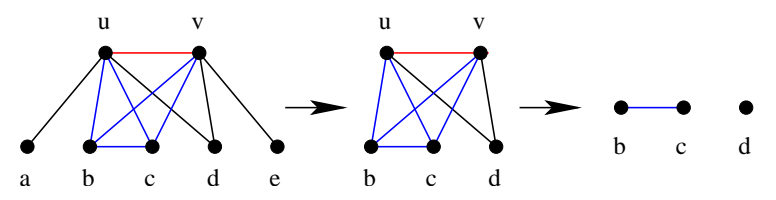

Figure 3: Given a graph $G=\left(V, E^{\prime}\right)$, we are looking for a largest clique containing the edge $\{u, v\}$. This clique is necessarily contained in the subgraph induced by $N(u) \cup N(v) \cup\{u, v\}=$ $\{b, c, d, u, v\}$. It is actually sufficient to compute the largest clique $\mathcal{C}$ in the subgraph induced by $N(u) \cap N(v)=N(u, v)=\{b, c, d\}$ since the clique we are looking for is nothing but $\mathcal{C} \cup\{u, v\}$ which, in our case, gives $\{u, v, b, c\}$
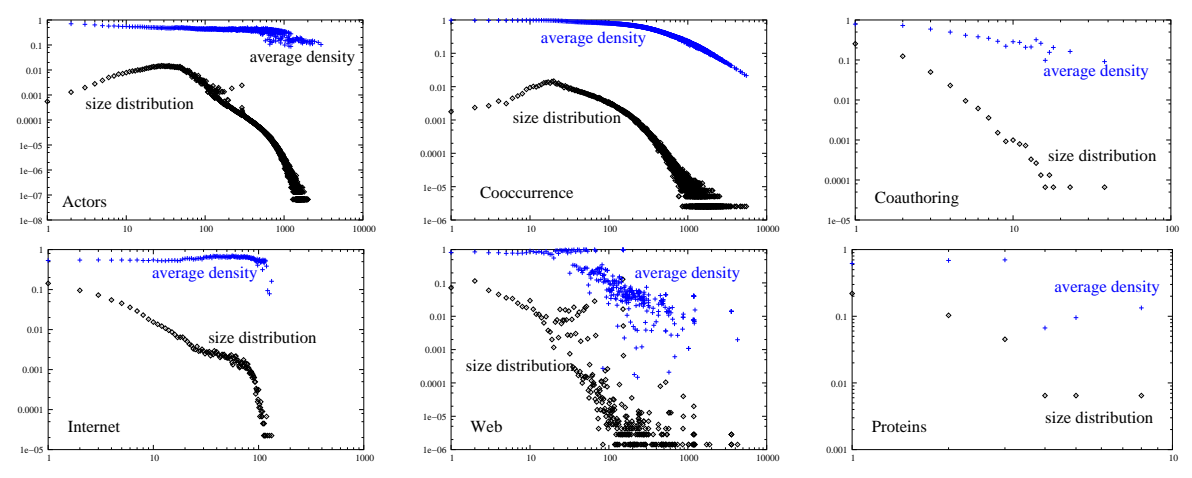

Figure 4: Distribution of the $N(u, v)$ sizes for all edges $(u, v)$, and average density of neighborhoods of given size.

\section{Properties of the bipartite graphs}

Given the general decomposition scheme, we can now transform any complex network into a bipartite graph. Figure 5 shows the top and bottom degree distribution for the natural bipartite networks Actors, Cooccurrence and Coauthoring, and the ones obtained for Internet, $W e b$ and Proteins graphs using our decomposition scheme.

All these distributions have a property in common: bottom degree distributions fit very well power laws in all cases. On the contrary, the top degree distributions are of two kinds: while Cooccurrence, Coauthoring, Internet and Proteins ones exhibit a Poisson behavior, Actors and $W e b$ ones are more heavy tailed.

These results lead to several remarks. First, the presence of a power law bottom degree distribution seems universal, just like the power law distribution in the $\perp$-projection of these graphs, but the effective exponent is not and depends on the considered complex network. Second, the top degree distributions can be qualitatively different and this point is important in the use of the bipartite structure for modeling complex networks since it can impact on some characteristics of the generated graphs. Further remarks will be pointed out in Section 6 .

Note also that the degree of a vertex in the $\perp$-projection is the sum of the degrees of the top vertices to which it is connected in the bipartite graph, minus the number of vertices in common in the neighborhood of these vertices. One can easily be convinced that this overlap between neighborhoods, if any, can have a great impact on the degree distribution. To deepen this notion of overlap, one can observe the correlation between the bottom vertex degrees in 

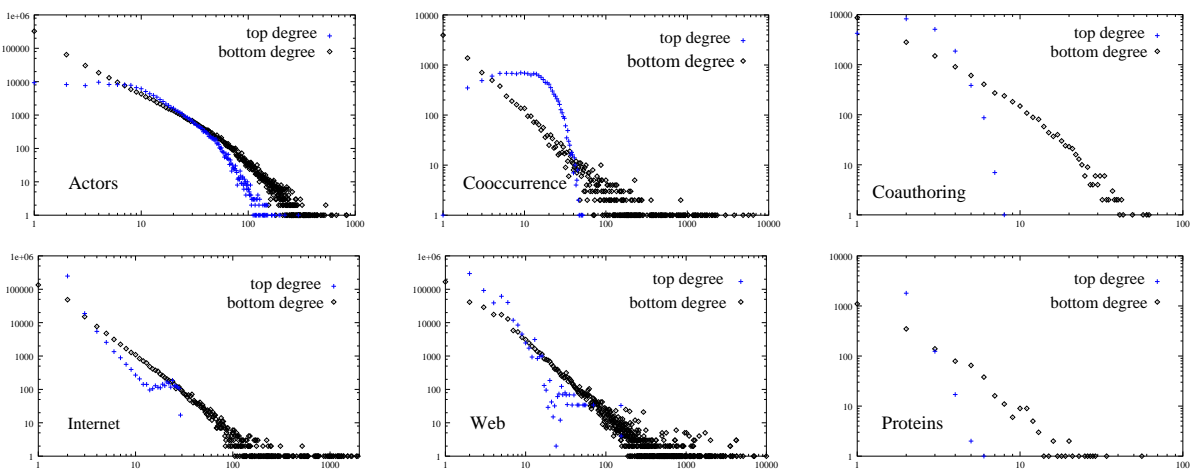

Figure 5: Top and bottom degree distributions for the natural bipartite versions of Actors, Cooccurrence, and Coauthoring, and for the bipartite version of Internet, Web, and Proteins obtained with the decomposition scheme.

both bipartite and $\perp$-projection (Figure 6). There exists nontrivial correlations in both cases, which are particularily strong in the case of Cooccurrence. Others remarks on the overlap will be discussed further in Section 6 .
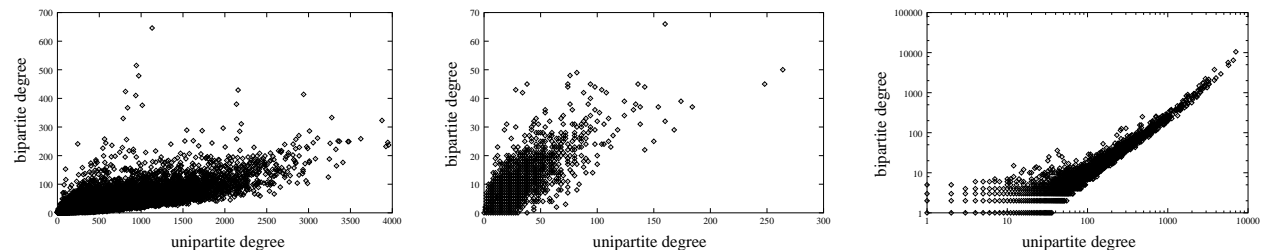

Figure 6: Correlations between degrees in the classical network and the bipartite one. From left to right: Actors, Coauthoring, and Cooccurrence.

Finally, we have shown in this section that all complex networks have a nontrivial underlying bipartite structure, which can be computed using our decomposition scheme. This leads us to the following question: is it possible to see the main properties of real-world complex networks as consequences of their underlying bipartite structure? We answer this question in the next sections.

\section{The bipartite models.}

Our aim is now to use the new general property of real-world complex networks discovered in the previous section, namely their underlying bipartite structure, as a way to propose a model which captures the main wanted properties.

As discussed in the first section of this paper, there are basically two ways to achieve this goal. First, we may try to sample random bipartite graphs with prescribed (top and bottom) degree distributions. Second, we may try to propose a construction process similar to the ones observed in practice, to obtain a growing model. 
We proposed such models in [34]. In order to deepen the understanding of these models, we here recall and discuss more precisely their definitions, and we provide a full (both analytic and experimental) analysis in the next sections.

\section{Random sampling of bipartite graphs with prescribed degree distribution}

One can sample uniformly a random bipartite graph with prescribed (top and bottom) degree distributions in the spirit of the configuration model as follows (see Figure 7) [17, 55, 56]:

1. generate both top and bottom vertices and assign to each vertex a degree drawn from the given distributions,

2. create for each vertex as many connection points as its degree,

3. link top and bottom connection points randomly,

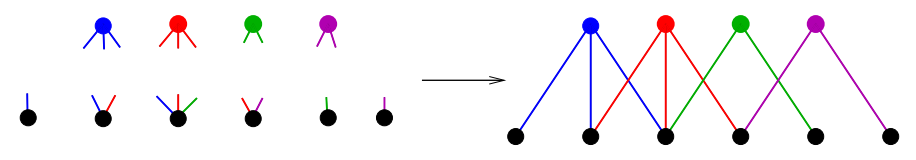

Figure 7: Construction of a random bipartite graph with prescribed degree distributions: first top and bottom vertices are drawn and each vertex is assigned a degree with respect to the given distributions, then edges are chosen randomly between the two sets.

This process generates random bipartite graphs uniformly within the set of bipartite graphs with the given degree distributions. However it cannot be used without taking care of the following constraints: top and bottom distributions cannot be arbitrary since they must allow the total degree of both sets to be equal. Actually one only has to ensure that the number of vertices times the mean of the distribution brings the same value for top and bottom sets. The second problem arises with the fact that even if the two distributions are theoretically consistent, two sets of degrees experimentally drawn from these distributions can be inconsistent (the sums of the degrees are different). A classical trick, which induces no bias, consists in dropping one top and one bottom vertex at random and redraw their degree [55, 56]. This last step may have to be done more than once before one obtains correct values but finally the implementation and use of the model is very simple and efficient [17.

Note that, just like with the MR model, multiple edges may appear. Again, one can easily show that they can be neglected when the graph is large. Moreover, some approaches exist [38, 44] which can easily be modified to obtain random bipartite graphs without multiples edges. This is however out of the scope of this paper.

\section{Growing bipartite model with preferential attachment}

The random bipartite model assumes that two distributions, for both top and bottom degrees, are explicitly given. One can also use other rules (preferential attachment for instance) to define them implicitly and introduce a growing model. Indeed, as already noticed, the bottom degree distributions follow a power law. This leads to the following model: at each step, a new top vertex is added and its degree $d$ is sampled from a prescribed (top) distribution (which qualitatively varies between graphs). Then, for each of the $d$ edges of the new vertex, 
either a new bottom vertex is added (with probability $1-\lambda$ ) or one is picked among the preexisting ones using preferential attachment (with probability $\lambda$ ). The parameter $\lambda$ is the overlap ratio, defined as the average ratio of preexisting bottom vertices to which a new top vertex is connected.

It is generally not possible to know exactly the order in which cliques are created on realworld bipartite graphs, but the average ratio can be computed globally as $\lambda=1-\frac{|\perp|}{\sum d_{\top}}$. One can compute it and get 0.733 for Actors, 0.877 for Coauthoring and 0.949 for Cooccurrence. Notice that $1-\lambda$ can be rewritten and is simply the inverse average bottom degree (since $\sum d_{\top}=\sum d_{\perp}$ ), therefore a high overlap ratio yields a high average bottom degree (since only few nodes are created at each time step).

At each step of the construction process, the bipartite graph has the required degree distributions: the prescribed top degree distribution is obtained by construction while the power law degree distribution is obtained using preferential attachment, which can be shown formally in exactly the same way as in the original AB model [3]. Notice moreover that this construction process is very similar to the one observed in some real-world cases. For instance, Actors is built exactly this way: when a new movie is produced (which corresponds to the addition of a top vertex), it is linked to actors according to their popularity, and to some new actors, playing in a movie for the first time.

We finally have two models to produce bipartite networks similar to the ones obtained from real-world complex networks, in terms of top and bottom degree distributions. The next question is to ask if they capture the other properties of interest in their $\perp$-projection, namely the average distance, the degree distribution and the clustering. We will answer positively to this question with formal arguments and with experimental results in the next sections.

\section{Analysis of the models.}

Our aim in this section is to give formal proofs for the main properties of the $\perp$-projection of a random bipartite graph with prescribed degree distributions. Some of these properties, and others, have been studied independently in [55, 56] with different techniques and a different point of view. We however believe that our proofs give new insight on these properties, therefore we give them below. In particular, our proof techniques may be considered as more mathematically rigorous.

Since these properties are induced by a typical graph (this is what random sampling gives us), this is a way to answer the following question: what properties are induced by the underlying bipartite structure? In particular, can we see the main properties of realworld complex networks, namely low average distance, power law degree distribution and high clustering, as consequences of the underlying bipartite structure?

We will see that it is indeed the case. Notice that many other properties, like the size distribution of the connected components for instance, are of high interest. It is shown in 55. that under reasonable conditions on the degree distributions the $\perp$-projection is connected, or at least has a giant component. In all the practical cases, these conditions are fulfilled, therefore we will restrict ourselves to this case. 


\section{Degree distribution}

Let us first consider the degree distribution of the $\perp$-projection of a random bipartite graph $G=(\top, \perp, E)$. Given a bottom vertex $u$, we denote by $d(u)$ the degree of $u$ in the bipartite graph, and by $d_{U}(u)$ its degree in its $\perp$-projection. We want to study the distribution of $d_{U}(u)$ (we actually deal here with the expected value for a randomly chosen $u$ ).

Lemma 1 Let us consider a bottom vertex $u \in \perp$. The expected number of bottom vertices which have a neighbor (in $\top$ ) in common with $u$, i.e. $d_{U}(u)$, is:

$$
\frac{d(u)}{|\top|} \cdot \sum_{t \neq u} d(t)+\mathcal{O}\left(\frac{d(u)^{2}}{|\top|^{2}} \cdot \sum_{t \neq u} d(t)^{2}\right)
$$

Proof. The exact expected value of $d_{U}(u)$ is given by $d_{U}(u)=\sum_{t \neq u}\left(1-\frac{\left(\begin{array}{c}|\top|-d(u) \\ d(t)\end{array}\right)}{\left(\begin{array}{l}\mid T(t) \\ d(t)\end{array}\right)}\right)$ since the probability that a given bottom vertex $t$ has a top neighbor in common with $u$ depends only on the degree of both vertices and the number of top vertices. To simplify this formula, we can approximate the ratio $\left(\begin{array}{c}|\mathrm{T}|-d(u) \\ d(t)\end{array}\right) /\left(\begin{array}{c}|\mathrm{T}| \\ d(t)\end{array}\right)$ as follows: $\frac{\left(\begin{array}{c}|\mathrm{T}|-d(u) \\ d(t)\end{array}\right)}{\left(\begin{array}{l}\mathrm{TI} \mid \\ d(t)\end{array}\right)}=\frac{(|\mathrm{T}|-d(u)) !(|\mathrm{T}|-d(t)) !}{|\mathrm{T}| !(|\mathrm{T}|-d(u)-d(t)) !} \sim$ $\frac{(|\mathrm{T}|-d(t))^{d(u)}}{|\mathrm{T}|^{d(u)}} \sim 1-\frac{d(t) d(u)}{|\mathrm{T}|}+\mathcal{O}\left(\left(\frac{d(t) d(u)}{|\mathrm{T}|}\right)^{2}\right)$.

Therefore: $d_{\top}(u) \sim \sum_{t \neq u}\left(\frac{d(t) d(u)}{|T|}+\mathcal{O}\left(\left(\frac{d(t) d(u)}{|T|}\right)^{2}\right)\right) \sim \frac{d(u)}{|T|} \sum_{t \neq u} d(t)+\mathcal{O}\left(\frac{d(u)^{2}}{\left.T \top\right|^{2}} \sum_{t \neq u} d(t)^{2}\right)$, which is the formula of the claim.

This lemma makes it possible to compute the probability for a vertex $u$ in the $\perp$-projection graph to have a given degree $k$ if the bottom degree distribution is a power law with exponent $\beta$ :

$$
P\left[d_{U}(u)=k\right] \sim P\left[d(u)=\frac{n}{\sum_{t \neq u} d(t)} \cdot k\right] \sim \frac{1}{\left.\left(\sum_{t \neq u} d(t)\right) \cdot k\right)^{\beta}} \sim k^{-\beta}
$$

Therefore, as long as the bottom degree distribution follows a power law, the degree distribution in the $\perp$-projection of the graph also follows a power law with the same exponent, which is indeed the case in practice as one can check in Figures 8 and 5 . The main reason comes from the fact that $\perp$-vertices are somehow randomly linked to $T$-vertices and their degree in the projection is therefore strongly correlated with their bipartire degree.

\section{Average distance}

To study the average distance in the $\perp$-projection of a graph obtained with the model, we will use a result from L. Lu about the diameter (i.e. the largest distance between any two vertices) of some specific random graphs:

Theorem 1 [39] Let $G=(V, E)$ be a graph whose vertices are weighted with weights $w_{1}, \cdots, w_{n}$, such that each edge $\{i, j\}$ appears with probability $w_{i} \cdot w_{j} \cdot p$. If the degrees of the vertices in $V$ follow a power law with an exponent $\beta$ strictly greater than 2 , then the diameter of the graph $G$ is almost surely $\Theta(\log (n)) \sqrt{5}$.

\footnotetext{
${ }^{5} f=\Theta(g)$ if and only if $f=\mathcal{O}(g)$ and $g=\mathcal{O}(f)$ i.
} 
This theorem, together with the one presented above on the degree distribution of the $\perp$-projection of the graph, leads to the following result:

Theorem 2 Let $G=(\top, \perp, E)$ be a bipartite graph such that the bottom degree distribution follows a power law with an exponent greater than 2 . Then the diameter of the $\perp$-projection of $G$ is almost surely $\Theta(\log (|\perp|))$.

Proof. Given two bottom vertices $u$ and $v$ in $\perp$, the probability that they are connected in the $\perp$-projection is equal to the probability that they are both linked to a same top vertex in $G$. This probability is exactly proportional to $d_{\perp}(u) \cdot d_{\perp}(v)$. Therefore we can apply Theorem 1 considering that the weight of each vertex is its degree and so the connection probability is ensured, and as long as bottom degree distribution follows a power law with an exponent $\beta$ strictly greater than 2 . The diameter of the $\perp$-projection of the graph therefore is almost surely $\Theta(\log (|\perp|))$.

Since the diameter is an upper bound for the average distance, this theorem implies that the average distance of the $\perp$-projection scales at most as fast as the logarithm of its number of nodes. Notice that, as in the case of random networks [13, 18, 21, 28, 39, 55, 56], the average distance may grow even slower.

\section{Clustering}

Recall that the clustering of a vertex $v$ of degree at least 2 in a graph is the probability that two of its neighbors are linked [70, i.e. the number of triangles to which $v$ belongs over the number of connected triples centered on it: $c(v)=\frac{|\triangle(v)|}{|\wedge(v)|}$. Then the clustering of the graph is defined as: $\frac{1}{N} \sum_{v, d(v)>1} c(v)$. We define the clustering of a vertex restricted to a part of its neighborhood as its clustering in the subgraph induced by this part of its neighborhood.

Hereafter we give a lower bound for the clustering of a graph $G^{\prime}$ which is the $\perp$-projection of a bipartite graph $G=(\top, \perp, E)$ obtained using the random bipartite model. We show that, under reasonable assumptions on the top and bottom degree distributions, it is bounded by a value independent of the size of the graph. This shows that the model produces graphs with nontrivial clustering.

Before entering in the core of this section, note that an approximation formula for the clustering of such a graph is given in [55, 56]. Here we give an exact formula for a lower bound. Both are interesting since the first one gives an expected value which is indeed very close to the real value, while the second one gives a guaranty that the exact value is above the given quantity. We used this approach because we seek qualitative results only, and so it is sufficient for us to show that the clustering does not tend to 0 when the size of the graph grows. The lower bound achieves this goal.

First, the probability for two top vertices to have more than one bottom vertex in common in their neighborhood tends to zero when the size of the graph grows. We therefore consider any vertex $b$ in the $\perp$-projection of the graph and we suppose that its neighborhood is composed of a set of disjoint cliques. We will prove the following:

- the effect of the number of top vertices of degree 2 to which $b$ is connected on its clustering is negligible, and 
- the clustering of $b$ can be bounded by a value which depends only on its degree.

Lemma 2 Let $T_{>2}$ denote the set of top neighbors of $b$ in $G$ with degree strictly greater than 2 , and $\perp_{>2}$ denote the set of bottom neighbors of $T_{>2}$. Let $p$ be the fraction of neighbors of $b$ which belong to $\perp_{>2}$, and $\alpha$ be the clustering of $b$ (in $G^{\prime}$ ) restricted to $\perp_{>2}$.

Then the clustering of $b$ in $G^{\prime}$ scales as $p^{2} \cdot \alpha$.

Proof. The fact that the clustering of $b$ restricted to $\perp_{>2}$ is $\alpha$ implies that $\left|\triangle_{\perp_{>2}}(b)\right|=$ $\alpha \cdot\left(\begin{array}{c}p \cdot d \\ 2\end{array}\right)$. If we consider the whole neighborhood of $b$, instead of just $\perp_{>2}$, the number of triangles does not change while the number of connected triples increases: $c(b)=\frac{\alpha \cdot\left(\begin{array}{c}p \cdot d \\ 2\end{array}\right)}{\left(\begin{array}{c}d \\ 2\end{array}\right)}=$ $\alpha \cdot \frac{p \cdot d((p \cdot d-1)}{d(d-1)} \sim p^{2} \cdot \alpha$ which is the formula of the claim.

Therefore, as long as $p$ is a constant, one can neglect the top vertices of degree 2 when computing the clustering of a given vertex. Now let us prove that the clustering of a bottom vertex can be related to its degree.

Lemma 3 If $b$ is connected only to top vertices of degree at least 3 in $G$, then:

$$
c(b) \geq \frac{1}{2 \cdot d(b)-1}
$$

Proof. Suppose that $b$ is connected to two top vertices, $t_{1}$ and $t_{2}$, of degree at least 3 (we deal with the general case below). Then the clustering of $b$ is $c(b)=\frac{\left.\frac{\left(d\left(t_{1}\right)-1\right.}{2}\right)+\left(\begin{array}{l}d\left(t_{2}\right)-1 \\ 2\end{array}\right)}{\left(\begin{array}{l}d\left(t_{1}\right)+d\left(t_{2}\right)-2 \\ 2\end{array}\right)}$ Suppose now that $b$ is connected to $t_{2}$ and $t_{1}^{\prime}$ such that $d\left(t_{1}^{\prime}\right)=d\left(t_{1}\right)+1$, then the clustering of $b$ is $c^{\prime}(b)=\frac{\left(\begin{array}{l}d\left(t_{1}\right)+1-1 \\ 2\end{array}\right)+\left(\begin{array}{l}d\left(t_{2}\right)-1 \\ 2\end{array}\right)}{\left(\begin{array}{c}d\left(t_{1}\right)+d\left(t_{2}\right)-1 \\ 2\end{array}\right)}$ and $c^{\prime}(b)-c(b)=\frac{2 \cdot\left(d\left(t_{2}\right)-1\right)}{\left(d\left(t_{1}\right)+d\left(t_{2}\right)-2\right) \cdot\left(d\left(t_{1}\right)+d\left(t_{2}\right)-3\right)}>0$, which means that the clustering grows with the degree of $t_{1}$ and $t_{2}$. A lower bound for the clustering of $b$ can therefore be obtained when both $t_{1}$ and $t_{2}$ have the smallest possible degree: 3 .

This can be extended to the case where $b$ has more than two top neighbors to obtain the

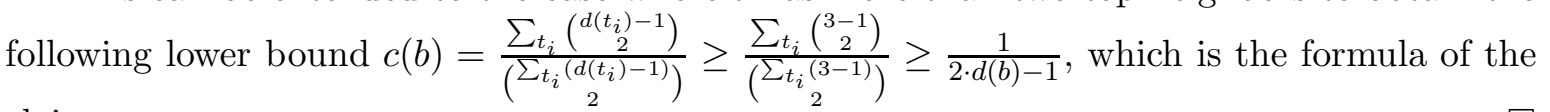
claim.

The clustering of the $\perp$-projection $G^{\prime}$ can now be easily approximated:

$$
c\left(G^{\prime}\right) \sim \frac{1}{N} \sum_{b \in \perp} \frac{1}{2 d(b)-1}
$$

As long as there is a linear number $c \cdot N$ of vertices $b$ of degree 2, the sum scales linearly with $N: \sum_{b \in \perp} \frac{1}{2 \cdot d(b)-1} \geq \sum_{b, d(b)=2}\left(\frac{1}{2 \cdot 2-1}\right)=\frac{c \cdot N}{3}$ (we could have considered vertices of any constant degree $k$ instead of 2). Therefore the lower bound for the clustering is independent of $N$. This holds in particular for power law networks since the number of vertices of degree 2 is of the order of $N \cdot 2^{-\alpha}$.

Since we do not consider top vertices of degree 2 in the last formula (due to Lemma 2), we must also ensure that the number of such top neighbors represent at most a constant fraction 
(not tending to 1) of the neighbors. This is indeed the case for most distributions and in particular for the ones met in practice. We finally obtain that the clustering of the graph is larger than a non-zero constant independently of the size of the graph. The idea behind the proof is that each node belongs to a small number of cliques and even if these cliques are disjoint, the clustering is still high.

\section{$5 \quad$ Experimental results}

The formal results of the previous section give a precise intuition on how the random bipartite graph model with prescribed degree distributions behaves. We can also check its properties experimentally by generating graphs using this model and the same parameters as the ones measured on real-world complex networks. This is what we do in this section with our six examples, for the purely random bipartite model as well as for the one with preferential attachment.

More precisely, the networks are generated using the models presented in section 3 with $\top$ and $\perp$ distributions obtained either from the real graphs when they are known or from the decomposition scheme introduced in section 2 ,

Table 3 and 4 give the values obtained for the average distance and the clustering. Figure 8 shows a comparison between the degree distributions of the original graphs, and the ones obtained with the two bipartite models.

\begin{tabular}{|l|c|c|c|c|c|c|}
\hline & Internet & Web & Actors & Co-auth & Co-occur & Protein \\
\hline \hline$d$ & 5.80 & 7 & 3.6 & 7.18 & 2.13 & 6.74 \\
\hline$d_{r b}$ & 2.97 & 3.2 & 3.06 & 5.07 & 2.06 & 5.8 \\
\hline$d_{g b}$ & 2.81 & 3.53 & 2.83 & 3.98 & 2.6 & 5.45 \\
\hline
\end{tabular}

Table 3: Average distance of the commonly used models and the bipartite models. For each network, we give its actual average distance, and the one obtained with the random bipartite model with prescribed degree distributions $d_{r b}$, and the growing one with preferential attachment $d_{g b}$.

\begin{tabular}{|l|c|c|c|c|c|c|}
\hline & Internet & Web & Actors & Co-auth & Co-occur & Protein \\
\hline \hline$c$ & 0.171 & 0.466 & 0.785 & 0.638 & 0.822 & 0.153 \\
\hline$c_{r b}$ & 0.32 & 0.663 & 0.767 & 0.542 & 0.831 & 0.187 \\
\hline$c_{g b}$ & 0.65 & 0.708 & 0.793 & 0.632 & 0.768 & 0.244 \\
\hline \hline
\end{tabular}

Table 4: Clustering obtained with the commonly used models and the bipartite models. For each network, we give its actual clustering and the clustering obtained with the random bipartite model with prescribed degree distributions $c_{r b}$, and the growing one with preferential attachment $c_{g b}$.

As expected from the previous section, the graphs obtained with the random bipartite model have a power law distribution of degrees, a small average distance and a high clustering. Moreover, by definition, they have the same distribution of cliques size as the original network. Therefore the model is qualitatively accurate for the modeling of general real-world complex networks: the simulations fit real-world values qualitatively well for both clustering and average distance, which proves the relevance of the underlying bipartite structure as an essential property to characterize real-world complex networks. 

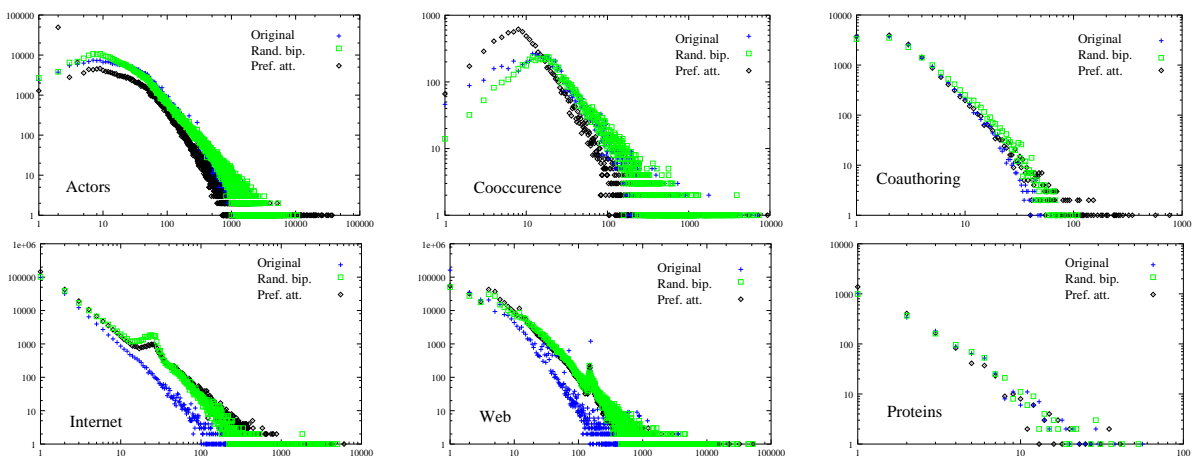

Figure 8: The original degree distribution of our six examples, together with the ones obtained with the random bipartite model and with the growing bipartite model.

There are however differences between the values obtained from the bipartite models and real-world networks. They are consequences of the following fact: in the original bipartite networks (both natural ones and the ones obtained from the decomposition), many top nodes have a large neighborhood intersection. In other words, the overlap between cliques is large (if two cliques have one neighbor in common, they certainly have many). This behavior can be viewed as a bipartite clustering and is not captured by the bipartite models. The random linking implies that most cliques have only one vertex in common, if any. This is responsible for both the inaccuracy of the models concerning some clusterings and for the irregularities one can observe on some distributions. Figure 9 plots the distribution of the overlap between cliques. This overlap is very small for all random bipartite graphs while it is non trivial for the original graphs.
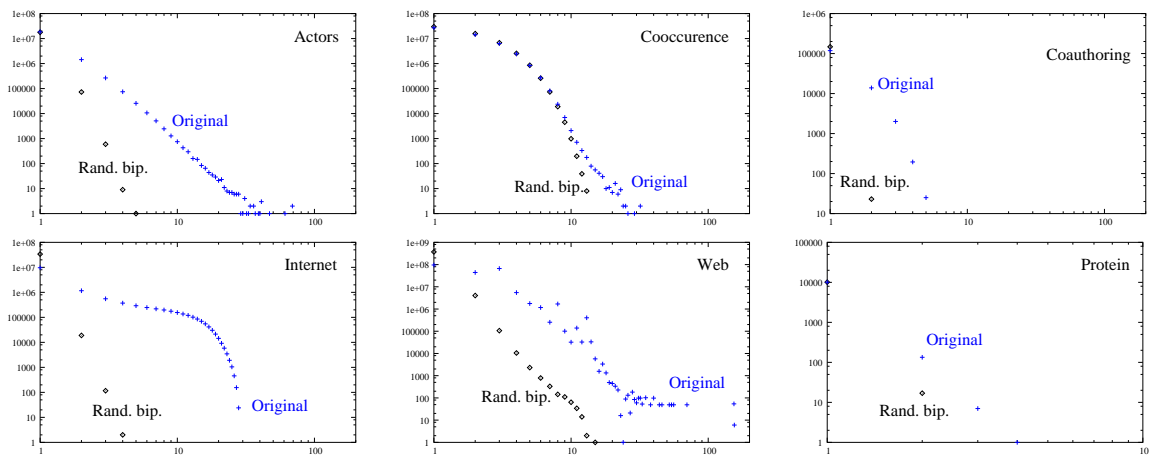

Figure 9: Distribution of the size of the overlap between cliques (i.e. intersections of neighbors of top nodes) for both original networks (or decomposed ones) and random bipartite ones.

More precisely, in the case of Internet, we noticed the presence of a sub-graph of only 94 vertices which contains all the 494 cliques of size 14 and more. This very dense sub-graph implies that the clustering of each of the 94 nodes is very high. However, they have almost no impact on the clustering of the whole graph (due to the average). On the other hand, in the $\perp$-projection of random bipartite networks, these large cliques are disseminated all over the graph which brings two artifacts: there are a lot of vertices having a degree between 14 and 
29 which explains the bump on degree distribution (a similar phenomenon can be observed on $W e b$ ), and the number of vertices with high clustering is drastically increased because of their presence in large cliques (from 94 to 50,000).

These experimental results should also be compared to the ones obtained with the currently most used models, presented in Section 1. This comparison gives evidence for the fact that the models we propose may be considered as an important step towards the realistic modeling of complex networks.

All these remarks hold both for the growing bipartite model and for the random one. This is worth to notice, since it may be very important in some contexts that the model produces growing graphs with realistic properties, and in other contexts that the obtained graphs are representative of a precise class of graphs.

\section{Conclusion and discussion}

In this paper, we propose bipartite graphs as a general tool for the modeling of real-world complex networks. They make it possible to achieve the following challenges:

- the obtained networks have the three main wanted properties (logarithmic average distance, high clustering and power law degree distribution),

- the models are based on a realistic construction process representative of what happens in some real-world cases, and

- their definitions are simple enough to make it possible to give some intuition and some proofs of their properties.

Moreover, they can be derived in two versions: one which relies on random sampling among a class of graphs, and one which relies on an iterative construction process. This makes them suitable for a wide variety of usages. Moreover, it is very simple to obtain graphs using this model (we provide a generator at [17]), which makes it highly suitable for simulation purposes.

The model is based on the discovery that all real-world complex networks have an underlying bipartite structure. Some networks naturally have this structure and, for the others, we show that they can be decomposed into cliques which make such a structure emerge. This shows that the main properties of complex networks can be viewed as consequences of this bipartite structure, and that the model captures a general behavior of complex systems.

However, as already stressed in previous sections, the overlapping between cliques is not taken into account by the bipartite model which in some way distributes cliques all over the networks independently of the nodes implied. On the contrary, it seems obvious that graphs such as Actors are not randomly constructed: actors from a same country are more likely to play together, for instance. This lack of overlapping can also be described on the bipartite graphs: if two top nodes have more than one bottom node in the intersection of their neighborhood, then this yields a non trivial bipartite clique. On the other hand, for the graphs generated with both bipartite models, most of these bipartite cliques are trivial ones (as long as there are no too many cliques).

An analogy can be made with the clustering in random graphs (ER graphs for instance), in which neighborhoods of vertices are very sparse while real-world neighborhoods are quite 
dense: one could say that real-world bipartite networks are bi-clusterized while random ones are not, even if they capture the most common properties.

There are many directions in which this work may be extended. Solving the previous drawback is one of them. This model might also be extended to the case of directed and weighted graphs. These problems rely on giving a new definition to the concept of clique which can be used in this context.

Another similar problem occurs when the graph is only partially known. In this case, some edges are missing, which might yield to only trivial cliques. A solution to this problem could be to study a model with quasi-cliques. Embedding this concept in the bipartite vision however is nontrivial and remains to be done.

One may also use this model to deepen the study of some phenomena of high interest like the robustness of networks, the spread of rumors and diseases, etc. The random graph model with prescribed degree distribution already led to important advances on these questions [19, 20, 52, 58. They should now be extended to the bipartite models in order to evaluate the impact of clustering on these problems. We argue that this is a strength of our approach since results on random graphs with prescribed degrees can be directly adapted to our model in order to take the clustering into account.

Finally, let us emphasize on the fact that the study of real-world complex networks is only at its beginning. The discovery of their statistical properties, the analysis of the impact of these properties, their integration into accurate models, and the use of these models in simulation and analysis are key issues for our understanding of real-world complex networks, which has crucial fundamental and applicative implications. Our work lies in this context. It proposes a solution to the problem of the realistic random modeling of real-world complex networks (in the sense of the three main observed properties), and it points out some relevant directions for further research.

Acknowledgments. We thank Annick Lesne, Clémence Magnien and James Martin for careful reading of preliminary versions and useful comments. We also thank the anonymous referees for helpful comments.

\section{References}

[1] J. Abello, P. Pardalos, and M. Resende. On maximum clique problems in very large graphs. External Memory Algorithms, DIMACS Series, AMS, 1999.

[2] W. Aiello, F.R.K. Chung, and L. Lu. A random graph model for massive graphs. In ACM Symposium on Theory of Computing (STOC), pages 171-180, 2000.

[3] R. Albert and A.-L. Barabási. Emergence of scaling in random networks. Science, 286:509-512, 1999.

[4] R. Albert and A.-L. Barabási. Statistical mechanics of complex networks. Reviews of Modern Physics 74, 47, 2002.

[5] R. Albert, H. Jeong, and A.-L. Barabási. Diameter of the world wide web. Nature, 401:130-131, 1999.

[6] R. Albert, H. Jeong, and A.-L. Barabási. Error and attack tolerance in complex networks. Nature, 406:378-382, 2000.

[7] arXiv.org e Print archive. http://arxiv.org/. 
[8] A.-L. Barabási, E. Ravasz, and T. Vicsek. Deterministic scale-free networks. Physica A 299, (3-4), pages 559-564, 2001.

[9] A. Barrat and R. Pastor-Satorras. Rate equation approach for correlations in growing network models. Phys. Rev. E., 71, 2005. 036127.

[10] E.A. Bender and E.R. Canfield. The asymptotic number of labeled graphs with given degree sequences. J. Combin. Theory Ser. A, 24:296-307, 1978.

[11] M. Boguna, R. Pastor-Satorras, and A. Vespignani. Epidemic spreading in complex networks with degree correlations. In al J.M. Rubi et, editor, XVIII Sitges Conference "Statistical Mechanics of Complex Networks". Springer Verlag, 2003.

[12] B. Bollobás. A probabilistic proof of an asymptotic formula for the number of labelled regular graphs. Europ. J Combinatorics, 1:311-316, 1980.

[13] B. Bollobás. Random Graphs. Academic Press, 1985.

[14] I. Bomze, M. Budinich, P. Pardalos, and M. Pelillo. The maximum clique problem. In D.-Z. Du and P. M. Pardalos, editors, Handbook of Combinatorial Optimization, volume 4. Kluwer Academic Publishers, Boston, MA, 1999.

[15] D.S. Callaway, M.E.J. Newman, S.H. Strogatz, and D.J. Watts. Network robustness and fragility: Percolation on random graphs. Phys. Rev. Lett., 85:5468-5471, 2000.

[16] Q. Chen, H. Chang, R. Govindan, S. Jamin, S. Shenker, and W. Willinger. The origin of power laws in internet topologies revisited. In INFOCOM, 2002.

[17] Source code for the random bipartite graph generator. http://jlguillaume.free.fr/www/programs.php.

[18] R. Cohen, D. ben Avraham, and S. Havlin. Handbook of graphs and networks, chapter 4: Structural properties of scale free networks. Wiley-VCH, 2002.

[19] R. Cohen, K. Erez, D. ben Avraham, and S. Havlin. Resilience of the internet to random breakdown. Phys. Rev. Lett., 85:4626-4628, 2000.

[20] R. Cohen, K. Erez, D. ben Avraham, and S. Havlin. Breakdown of the internet under intentional attack. Phys. Rev. Lett., 86:3682-3685, 2001.

[21] R. Cohen and S. Havlin. Scale free networks are ultrasmall. Phys. Rev. Lett., 90, 2003.

[22] F. Comellas, G. Fertin, and A. Raspaud. Vertex labeling and routing in recursive clique-trees, a new family of small-world scale-free graphs. In Sirocco 2003 - The 10th Int. Colloquium on Structural Information and Communication Complexity, pages 73-87.

[23] Self-Organized Networks Database. http://www.nd.edu/ networks/database/index.html.

[24] The Internet Movie Database. http://www.imdb.com/.

[25] S.N. Dorogovtsev and J.F.F. Mendes. Exactly solvable small-world network. Euro. phys. Lett., $50(1): 1-7,2000$.

[26] S.N. Dorogovtsev and J.F.F. Mendes. Evolution of networks. Adv. Phys. 51, 1079-1187, 2002.

[27] S.N. Dorogovtsev, J.F.F. Mendes, and A.N. Samukhin. Structure of growing networks with preferential linking. Phys. Rev. Lett. 85, pages 4633-4636, 2000.

[28] S.N. Dorogovtsev, J.F.F. Mendes, and A.N. Samukhin. Metric structure of random networks. Nucl. Phys. B 653, 307, 2003.

[29] P. Erdös and A. Rényi. On random graphs I. Publ. Math. Debrecen, 6:290-297, 1959.

[30] M. Faloutsos, P. Faloutsos, and C. Faloutsos. On power-law relationships of the internet topology. In SIGCOMM, pages 251-262, 1999. 
[31] R. Ferrer and R.V. Solé. The small-world of human language. In Proceedings of the Royal Society of London, volume B268, pages 2261-2265, 2001.

[32] Internet Maps from Mercator. http://www.isi.edu/div7/scan/mercator/maps.html.

[33] R. Govindan and H. Tangmunarunkit. Heuristics for internet map discovery. In IEEE INFOCOM 2000, pages 1371-1380, Tel Aviv, Israel, March 2000. IEEE.

[34] Jean-Loup Guillaume and Matthieu Latapy. Bipartite structure of all complex networks. Information Processing Letters (IPL), 90(5):215-221, 2004.

[35] H. Jeong, B. Tombor, R. Albert, Z. Oltvai, and A.-L. Barabási. The large-scale organization of metabolic networks. Nature, 407, 651, 2000.

[36] B.J. Kim, C.N. Yoon, S.K. Han, and H. Jeong. Path finding strategies in scale-free networks. Phys. Rev. E 65, 027103., 2002.

[37] R. Kumar, P. Raghavan, S. Rajagopalan, D. Sivakumar, A. Tomkins, and E. Upfal. Stochastic models for the web graph. Proceedings of the 41th IEEE Symp. on Foundations of Computer Science, 2000.

[38] M. Latapy and F. Viger. Random generation of large connected simple graphs with prescribed degree distribution. In proceedings of the 11-th international conference on Computing and Combinatorics COCOON'05, 2005.

[39] L. Lu. The diameter of random massive graphs. In ACM-SIAM, editor, 12th Ann. Symp. on Discrete Algorithms (SODA), pages 912-921, 2001.

[40] T. Luczak. Sparse random graphs with a given degree sequence, in Random Graphs, vol. 2. A.M. Frieze, T. Luczak eds. Wiley, New York, 1992. pages. 165-182.

[41] D. Magoni and J.-J. Pansiot. Influence of network topology on protocol simulation. In ICN'01 1st IEEE International Conference on Networking, volume Lecture Notes in Computer Science, pages 762-770, July 9-13, 2001.

[42] M. Mihail and C. Papadimitriou. the eigenvalue power law, 2002.

[43] S. Milgram. The small world problem. Psychology today, 1:61-67, 1967.

[44] R. Milo, N. Kashtan, S. Itzkovitz, M.E.J. Newman, and U. Alon. On the uniform generation of random graphs with prescribed degree sequences, 2003. cond-mat/0312028.

[45] M. Molloy and B. Reed. A critical point for random graphs with a given degree sequence. Random Structures and Algorithms, pages 161-179, 1995.

[46] M. Molloy and B. Reed. The size of the giant component of a random graph with a given degree sequence. Combin. Probab. Comput., pages 295-305, 1998.

[47] S.D. Monson, N.J. Pullman, and R. Rees. A survey of clique and biclique coverings and factorizations of (0,1)-matrices. Bull. Inst. Combin. Appl., 14:17-86, 1995.

[48] C. Moore and M.E.J. Newman. Epidemics and percolation in small-worlds networks. Phys. Rev. E, 61:5678-5682, 2000.

[49] Adilson E. Motter and Ying-Cheng Lai. Cascade-based attacks on complex networks. Physical Review E 66, 2002.

[50] M.E.J. Newman. Scientific collaboration networks: I. Network construction and fundamental results. Phys. Rev. E, 64, 2001.

[51] M.E.J. Newman. Scientific collaboration networks: II. Shortest paths, weighted networks, and centrality. Phys. Rev. E, 64, 2001.

[52] M.E.J. Newman. The spread of epidemic disease on networks. Phys. Rev. E, 66, 2002. 
[53] M.E.J. Newman. mixing patterns in networks. Phy. Rev. E, 67, 2003. cond-mat/0209450.

[54] M.E.J. Newman. The structure and function of complex networks. SIAM Review, 45(2):167-256, 2003.

[55] M.E.J. Newman, D.J. Watts, and S.H. Strogatz. Random graphs with arbitrary degree distributions and their applications. Phys. Rev. E, 2001.

[56] M.E.J. Newman, D.J. Watts, and S.H. Strogatz. Random graph models of social networks. Proc. Natl. Acad. Sci. USA, 99 (Suppl. 1):2566-2572, 2002.

[57] J. Orlin. Contentment in graph theory: Covering graphs with cliques. Indigationes Mathematicae, 80:406-424, 1977.

[58] R. Pastor-Satorras and A. Vespignani. Epidemic spreading in scale-free networks. Phys. Rev. Lett., 86:3200-3203, 2001.

[59] P.Holme and B.J. Kim. Growing scale-free networks with tunable clustering. Phys. Rev. E, 65, 2002. 026107.

[60] M.A. Serrano and M. Bogu nà. Tuning clustering in random networks with arbitrary degree distributions. Phys. Rev. E., 72, 2005. 036133.

[61] M.A. Serrano, M. Bogu nà, and A. Díaz Guilera. Competition and adaptation in an internet evolution model. Phys. Rev. Lett., 94, 2005. 038701.

[62] S.H. Strogatz. Exploring complex networks. Nature 410, 2001.

[63] Lakshminarayanan Subramanian, Sharad Agarwal, Jennifer Rexford, and Randy H. Katz. Characterizing the internet hierarchy from multiple vantage points. In Proc. of IEEE INFOCOM 2002, New York, NY, Jun 2002.

[64] H. Tangmunarunkit, R. Govindan, S. Jamin, S. Shenker, and W. Willinger. On characterizing network hierarchy. Technical Report 03-782, Computer Science Department, University of Southern California, 2001. submitted.

[65] A. Vazquez. Growing network with local rules: Preferential attachment, clustering hierarchy, and degree correlations. Phys. Rev. E., 67, 2003. 056104.

[66] A. Vazquez, A. Flammini, A. Maritan, and A. Vespignani. Modeling of protein interaction networks. ComplexUs 1, 38, 2003.

[67] Bible Today New International Version. http://www.tniv.info/bible/.

[68] T. Walsh. Search in a small world. In IJCAI, pages 1172-1177, 1999.

[69] B. Wang, H. Tang, Z. Zhang, and Z. Xiu. Evolving scale-free network model with tunable clustering. Int. J. Mod. Phys. B, 19(26):3951-3959, 2005.

[70] D.J. Watts and S.H. Strogatz. Collective dynamics of small-world networks. Nature, 393:440-442, 1998. 